GLOBAL RESEARCH ETHICS

\title{
Between universalism and relativism: a conceptual exploration of problems in formulating and applying international biomedical ethical guidelines
}

G B Tangwa

J Med Ethics 2004;30:63-67. doi: 10.1136/jme.2003.003194

In this paper, the author attempts to explore some of the problems connected with the formulation and application of international biomedical ethical guidelines, with particular reference to Africa. Recent attempts at revising and updating some international medical ethical guidelines have been bedevilled by intractable controversies and wrangling regarding both the content and formulation. From the vantage position of relative familiarity with both African and Western contexts, and the privilege of having been involved in the revision and updating of one of the international ethical guidelines, the author reflects broadly on these issues and attempts prescribing an approach from both the theoretical and practical angles liable to mitigate, if not completely eliminate, some of the problems and difficulties.

\section{Correspondence to: G B Tangwa, University of Yaounde 1, Yaounde, Cameroon; gbtangwa@yahoo.com}

Received 20 January 2003 Revised 27 October 2003 Accepted8December 2003
P roblems that arise with the formulation of biomedical ethical guidelines are mainly conceptual and theoretical, whereas those that arise with application of such guidelines are mainly practical and procedural. At the first level, there is the need to capture clearly in concepts and language an ethical imperative and, at the second level, there is the need to translate an ethical rule or recommendation into concrete action within a specific time and place.

To say that it is morally right or wrong to do or refrain from doing something is to imply that it is so under all other similar circumstances, irrespective of place, time, and sociocultural context. Moral norms/rules may, of course, be expressed in, mixed with, or reflected in laws, societal customs, cultural practices, taboos, etiquette, and so on. But all these differ from moral norms/rules proper in that they are-by their very nature and raison d'etre-context bound. A law, for instance, has no jurisdiction and no applicability outside of its area of sovereignty.

Moral precepts are necessarily universal as well as abstract and, if their dynamic and dialectical relation with concrete particulars is not properly appreciated, they may appear rather empty. ${ }^{1}$ Moral judgments or propositions are different from expedient and other such judgments in that they are "universalisable" or generalisable. Ethical demands, moreover, are uncompromising in a way that other demands, such as the economic, political, social, and legal are not. In these latter demands, a certain measure of expediency or inconsistency may be permissible or tolerable, whereas expediency or unjustifiable inconsistency is completely antithetical to morality.

Morality has a special status which is evident from consideration of the fact that, although there is no better reason for condemning or recommending abolition of a law, custom, social, or cultural practice other than that it is morally obnoxious, no violation of a moral injunction can be justified on such grounds as that it is required by the law, is social custom, or usual practice. But the uncompromising and universal nature of an ethical rule or judgment does not necessarily imply that it could never justifiably be violated, if the particular situation and circumstances so warrant. To a deranged potential murderer in pursuit of his potential victim, for example, it would be justifiable for any third party, such as a bystander, to lie, without any implication that lying is thereby not universally morally wrong. Immanuel Kant's great mind apparently failed him on this issue and he ended with a quite counterintuitive situation, according to which it would still be morally wrong to lie in the above circumstances. $^{2}$

The same point can be made by drawing a distinction between universalism and absolutism. ${ }^{3}$ Moral rules are universal but not absolute; they can admit justifiable exceptions. Such putative exceptions, however, do not justify the postmodernist relativistic position that moral judgments are or ought to be entirely culture bound or culture dependent. In other words, accepting the possibility of a justifiable exception to the applicability of a moral rule in no way implies moral relativity, let alone the absurd idea of "geographical morality" or ethics which change at territorial borders. ${ }^{5}$ Obeying or applying an ethical rule, however, is necessarily done within the constraints of the particular place, time, circumstances, and perspective.

To say that there are ethical universals - that is, norms and values having cross cultural validity - is to make an understatement. It would be more accurate to say that all ethical norms or rules are cultural universals, because a rule or

Abbreviations: CIOMS, Council for International Organisations of Medical Sciences; DoH, Declaration of Helsinki; NBAC, National Bioethics Advisory Commission; WMA, World Medical Association 
norm cannot properly be described as "ethical" unless it is understood as having cross cultural validity, in the sense of being perceived as applying in all similar circumstances, irrespective of place and time. ${ }^{6}$

\section{INTERNATIONAL ETHICAL GUIDELINES}

In recent years there has been an explosion in biomedical research activities in the developing world by developed world researchers, thanks to great improvements in medical technologies, research techniques, and research funding in the Western world. These activities have been accompanied in some instances by abuse and malpractice, not unlike those which historically led to ethical concern in medical research. An illustrative case is that of a clinical trial designed to test a drug called trovafloxacine, which was carried out on children in northern Nigeria in 2001 during a meningitis epidemic, and resulted in eleven deaths, while a further two hundred of the children became blind, deaf, or lame. ${ }^{78}$

Biomedical research in the developing world, especially in Africa, faces many challenges, dilemmas, and difficulties, including trying to conform with some of the regulations laid down in the international regulatory texts. In a community in which people do not keep any important secrets from one another, under pain of being suspected of having been initiated into witchcraft, for example, the idea of confidentiality — so much emphasised in Western biomedical ethics and in the international regulatory texts-is practically inoperative in its Western form and boils down essentially to trust and confidence ensuring reliability. In such a community, the sense of protection that confidentiality is supposed to give might be subverted by such metaphysical beliefs as in witchcraft or lack of familiarity and/or trust in researcher or research. In such a community or similar other, the idea of autonomy (again well reflected in the regulatory texts) may boil down essentially to respect for other human beings as moral equals.

Furthermore, in a situation of general poverty combined with a high burden of disease, as we have today in some parts of sub-Saharan Africa, there is no way to prevent victims or potential victims of a deadly epidemic such as HIV/AIDS from being unduly induced by any type of research participation proposal that holds out the possibility of any type of treatment. This situation is responsible, on the one hand, for what has been termed the "therapeutic fallacy" by which research and other investigative procedures may deceptively be presented as if they were therapeutic interventions and, on the other, for what I would call the "therapeutic illusion", by which research subjects continue to believe, even against all explanation to the contrary, that they are undergoing treatment. In such a situation, the prohibition of inducement, due or undue, has no practical applicability and, if strictly observed, would amount to a prohibition of all medical research, which may not be ethical or even rational under the circumstances.

Regulatory frameworks for ethics in medical research are necessary at several different levels. A visual model for such a system of regulations would look like concentric circles, with the widest circle representing well formulated international guidelines and the smallest circle delimiting the standard operating procedures of local or institutional ethics review committees.

\section{THE HELSINKI DECLARATION AND CIOMS GUIDELINES}

Among international ethical guidelines, pre-eminence is generally accorded to the Declaration of Helsinki (DoH) and the Council for Internation Organisations of Medical Sciences (CIOMS) International Ethical Guidelines for Biomedical Research Involving Human Subjects. These two documents have recently been revised and updated, the DoH in 2000 and the CIOMS guidelines in 2002.

In the introduction to the CIOMS Guidelines, it is clearly stated, among other things, that the guidelines are "...a logical development of the Declaration of Helsinki...intended to indicate how the ethical principles embodied in the Declaration could be effectively applied in developing countries".

When the new, revised, and updated Declaration of Helsinki was first presented to the public during an international conference organised for the purpose in Pretoria, South Africa, 27-28 March 2001, Dr Delon Human, Secretary General of the World Medical Association (WMA) explained the main motivations for the revision in the following terms:

"When we...realized that there was genuine confusion caused by some of the articles in the previous version, we embarked on a comprehensive, consultative revision process to try to improve the DoH. Here I refer to issues such as the distinction between therapeutic and non-therapeutic research, the meaning of the "best proven diagnostic and therapeutic method", the standard of care ethically required for participants in biomedical research and the use of placebos in research. ...we are confident that the additions to the DoH have strengthened the document. ${ }^{10}$

As for the CIOMS Guidelines, their main purpose, as stated in the "Background Note" of the 1993 edition, is:

"...to indicate how the ethical principles...as set forth in the Declaration of Helsinki, could be effectively applied, particularly in developing countries, given their socioeconomic circumstances, laws and regulations, and executive and administrative arrangements. ${ }^{11}$

The emphatic focus on the "developing world" is also to be found in other ethical documents, such as the United States National Bioethics Advisory Commission's (NBAC's) Ethical and Policy Issues in International Research: Clinical Trials in Developing Countries (2001) and the Nuffield Council on Bioethics' The Ethics of Research Related to Healthcare in Developing Countries (2002).

\section{THE CONTROVERSIAL GUIDELINES OR ARTICLES}

Of the 30 paragraphs of the newly revised version of the Declaration of Helsinki, controversy has centred on two, paragraphs 29 and 30:

(29) The benefits, risks, burdens and effectiveness of a new method should be tested against those of the best current prophylactic, diagnostic, and therapeutic methods. This does not exclude the use of placebo, or no treatment, in studies where no proven prophylactic, diagnostic or therapeutic method exists.

(30) At the conclusion of the study, every patient entered into the study should be assured of access to the best proven prophylactic, diagnostic and therapeutic methods identified by the study.

Looking at previous versions of the $\mathrm{DoH}$, it is interesting to notice the following:

(1) The seminal ideas of paragraphs 29 and 30, now separate guidelines in the 2000 version, were captured in a single guideline in the 1996 version, which read: "In any medical study, every patient-including those of a control group, if any-should be assured of the best proven diagnostic and therapeutic method. This does not exclude the use of inert placebo in studies where no proven diagnostic or therapeutic method exists."

(2) In the 1975 version, the second part of the guideline, referring to placebo, added in the 1996 revision, did not exist and the guideline simply read: "In any medical study, every patient-including those of a control group, if any-should 
be assured of the best proven diagnostic and therapeutic method."

(3) In the original 1964 Declaration, there was no mention at all of either placebo or standard of care.

The seminal idea of paragraph 30 (DoH 2000) can be seen as being in line with the fundamental mission of the Declaration to protect the life, health, and interest of human subjects of biomedical research. For over 25 years, it remained uncontested and unchanged. However in the period leading up to the 2000 revision, it was put under question by some very influential voices and a strong lobby seeking a relaxation/reinterpretation of its demands, so as to accommodate clinical trials and medical research in which less than the best treatment could be assured for participants. In fact, this lobby and the powerful influences and interests behind it seem to have been largely responsible for the decision to carry out the revision, which culminated in the 2000 version of Helsinki.

Crudely but succinctly put, the line of argument of those who wanted a revision of the guideline is that comparing a new intervention or product with what is less than the best treatment might be more efficient in demonstrating effectiveness, using fewer trials, and less time and money, and also that it does not make sense to offer the best available treatment to people in "resource poor" environments, whose health system can hardly sustain it, or who would subsequently not be able to afford it after the trials. The first and main part of this argument is a pragmatic argument based on scientific and economic considerations. The opposing point of view is well expressed by Carel Ilssemuiden, a researcher in South Africa: "For many health researchers working with and in poor communities and countries, the attempts at reducing the minimum requirements for treatment in control arms of studies came as an "unbelievable" development. Those who are not distanced from these communities by oceans or socioeconomic gaps are seriously concerned about the efforts to reduce the potential benefits of research involving these specific individuals and communities rather than, as current ethics would have dictated ...to strengthen the protective and distributive nature of health research in vulnerable populations." ${ }^{12}$

And, as Ilssemuiden had correctly remarked and predicted, the pressure to lower the ethical demand seems "massive and well resourced" and there is "a great likelihood that the current phrasing of paragraphs 29 and 30 will be very temporary indeed". ${ }^{13}$ This became very clear when, in the course of the same conference to which allusion has been made, some of the other presenters launched a severe attack on Helsinki 2000. The gist of their arguments was that the distinction made in the Declaration between therapeutic and non-therapeutic research is illogical; that the Declaration is out of touch with contemporary ethical thinking, especially in its position on placebo controls and that, for these reasons, the Declaration had been widely disregarded and had lost its authority and prestige.

The intractable controversy over these two paragraphs of the Declaration led to the publication by the WMA in 2002 of a "note of clarification" regarding paragraph 29, which appears to allow use of placebo in certain cases outside of the uncontroversial "no other treatment available" arena. In particular, it would seem to permit the use of placebo "for compelling and scientifically sound methodological reasons" so long as permanent damage is avoided and subjects are not subjected to more than transient discomfort. Another note of clarification as well as a proposed amended version of paragraph 30 is currently under discussion.

The revision of the CIOMS guidelines, in which I have been privileged to be involved, took place almost concurrently with those of the DoH. After the adoption of Helsinki 2000 and the controversies that ensued over paragraphs 29 and 30, this work of revision continued, inevitably perhaps, in the shadow of these controversies. A draft version of the revised guidelines placed on the internet in June 2001, for public appreciation and comment, attracted hundreds of comments and suggestions from both individuals and corporate bodies from all over the globe, particularly from the industrialised Western world. My recollection and general observation are that the more specific and detailed any guideline attempted to be, the more disagreement and controversy it elicited. CIOMS guideline 11 is a good case in point. In the evolution of its various formulations and reformulations, it is hardly controversial if, in each case, the guideline had been limited to only the first paragraph, where the ethical imperative underlying the guideline is stated in broad, general terms. The intractable controversies inevitably begin where there is an attempt to give too much specific detail.

CIOMS 11 is the only guideline which begins with a definition of terms and explanatory notes (a precedent followed in paragraph 30 of the DoH, currently under discussion). The first explanatory note to the penultimate draft version of CIOMS guideline 11 read as follows:

\section{Note 1 on guideline 11}

The text and commentary of draft Guideline 11 were revised at the CIOMS Guidelines Conference held in February/March 2002 and the revised text was intensively discussed. Nevertheless, although the revision was well received, disagreements persisted. Some participants expressed reservations about, or opposed, the ethical acceptability of the exception to the general rule limiting the use of placebo to the three conditions set out in the Guideline. They held that the exceptional use of a comparator other than an established effective intervention could be interpreted to permit exploitation of poor and disadvantaged populations. They advanced three arguments:

- Placebo control could expose research subjects to risk of serious or irreversible harm when the use of an established effective intervention as comparator could avoid the risk.

- Not all scientific experts agree about conditions under which an established effective intervention used as a comparator would not yield scientifically reliable results.

- An economic reason for the unavailability of an established effective intervention cannot justify a placebocontrolled study in a country of limited resources when it would be unethical to conduct a study with the same design in a population with general access to the effective intervention outside the study.

Proponents of the exception argued that the populations of the poorer countries were in desperate need of low cost, technologically appropriate, public health solutions to their burden of disease, and were therefore liable to exploitation. It was for the guidelines, however, to encourage research to find local solutions, while providing clear guidance on how to protect against exploitation. In the drive to find global solutions for the global marketplace there was a risk of ignoring the importance of the local context. The spirit of the guidelines was to promote essential, scientifically sound and ethical research that is responsive to the needs of diverse countries and communities.

The guideline itself read as follows:

\section{Guideline 11: choice of control in clinical trials}

As a general rule, research subjects in the control group of a trial of a diagnostic, therapeutic, or preventive intervention should receive an established effective intervention. In some circumstances it may be ethically acceptable to use an alternative comparator, such as placebo or "no treatment". 
Placebo may be used:

- when no proven intervention exists

- when withholding an established effective intervention would expose subjects to, at most, temporary discomfort or delay in relief of symptoms

- when use of an established effective intervention as comparator would not yield scientifically reliable results and use of placebo would not add any risk of serious or irreversible harm to the subjects.

An exception to the general rule is applicable in some studies designed to develop a therapeutic, preventive or diagnostic intervention for use in a country or community in which an established effective intervention is not available and unlikely in the foreseeable future to become available, usually for economic or logistic reasons. The purpose of such a study is to make available to the population of the country or community an effective alternative to an established effective intervention that is locally unavailable. Accordingly, the proposed investigational intervention must be responsive to the health needs of the population from which the research subjects are recruited and there must be assurance that, if it proves to be safe and effective, it will be made reasonably available to that population. Also, the scientific and ethical review committees must be satisfied that the established effective intervention cannot be used as comparator because its use would not yield scientifically reliable results that would be relevant to the health needs of the study population. In these circumstances an ethical review committee can approve a clinical trial in which the comparator is other than an established effective intervention, such as placebo or no treatment or a local remedy.

At a CIOMS meeting in February-March 2002, the above guideline might unanimously have been approved if the last paragraph outlining an exception had simply been deleted, but its proponents were too tenacious and well placed to prevent its deletion. That the guideline does not appear as above in the final approved version of the revised guidelines is thanks to a last minute proposal by a member of the CIOMS Executive Committee, which fortuitously got accepted. In sum, the proposal suggested that, given the highly controversial nature of the "exception" to the general rule for the ethical use of placebos, it was inappropriate to leave the paragraph allowing for the exception as part of the guideline itself; the paragraph should instead be moved to the commentary on the guideline-along with the objections already stated in the Note originally proposed for the preface or introduction to the guidelines.

\section{MY OWN PRESCRIPTION}

The above proposal is substantially what we now have as Guideline 11. The controversial "exception" has been pushed to the commentary on the guideline, where the main arguments for or against the exception have also been stated. Although the debate might seem inconclusive either way there is the worry that, although the exception could possibly be justifiable (like lying) under particular circumstances, such putative particular circumstances cannot be specified in advance, a priori, in ethical guidelines of this type without the probability of turning them into de facto self fulfilling prophecies. However, the inclusion of the arguments of both sides of the controversy in the commentary is a highly commendable development, which both shows a way out of an impasse and preserves the thinking of earnest deliberators at a particular point in time for future appreciation and critical appraisal.

My personal hope is that CIOMS Guideline 11 , when it is next revised, will discard the idea of "ethically exceptional use of placebo control", which in my view places purely scientific and economic considerations ahead of ethical considerations, contrary to Helsinki paragraph 5 which unequivocally-in line with both reason and ordinary common sense-requires that the interests of the human subject of biomedical research be placed above the interests of both science and society. Furthermore, the reasoning supporting the exception appears to me to be in the nature of rationalisation for violations of an ethical imperative on the basis of transient contingent facts.

International ethical guidelines should be guidelines, a framework for guiding particular actions, and not detailed ready rules of thumb. As Benatar and Singer have rightly remarked, guidelines are like constitutions that require interpretation. ${ }^{14}$ No constitution can possibly incorporate its own comprehensive interpretation. No ethical guideline can possibly list all the putative exceptions to itself and, that apart, it is odd for any rule to seek to include the acceptable conditions of its own violation.

Biomedical research rules of thumb are best elaborated at the local rather than international level. To the extent that any of the guidelines expresses a genuine ethical imperative, to that same extent would it be universally relevant and applicable. But to apply it in a particular concrete situation, it must necessarily be shaped and coloured, like water in a container, by all the data furnished by particular context and perspective. To attempt determining such details for one milieu from another milieu is to run the high risk of serious error. In any case no such details, determined a priori, can provide advance justification for violation of ethical imperatives without prior in situ contextual appreciation of all the particularising, constraining, or compelling concrete circumstances. For those who seek ready rules of thumb that can be applied thoughtlessly with a guarantee of exoneration from blame, no set of truly international ethical guidelines could ever be formulated. To attempt formulating such rules for the international community-given its immense cultural diversity and situational differentiation-is to distrust and to undermine the rational ability and/or moral sensitivity of some human beings in appreciating and acting in accordance with moral imperatives, norms, or rules.

\section{CONCLUSION}

International ethical guidelines need to be formulated, not only in general as opposed to particularistic terms but in such general terms as would make sense and meaning to variously and differently situated and circumstanced human communities, groups, and assemblages. This is not a task that is likely to be adequately accomplished by one conceptually or ideologically homogenous group of human beings, no matter how altruistically minded, no matter how well intentioned and well equipped it may be, for and on behalf of the heterogeneous all. What a good international ethical guideline requires in its formulation and expression is a balancing of different but not necessarily conflicting points of view and perspectives-the underlying ethical imperative alone remaining constant. So formulated, its practical application would already be easier, to the extent that the ethical imperative is easily comprehended, now requiring only interpretation and translation into the idioms, expressions, practices, and manner of doing within any given community or locality. That, however, is not to say that any such putative guideline could ever unquestioningly be accepted everywhere at all times without controversy. That is a luxury not given to ethical judgments or discourse. However, in ethics correct action is more important than correct language.

Much of the difficulty with controversial ethical guidelines, from my point of view, arises from confusing or presenting cultural particulars as universals, or from too much emphasis 
and concentration on the mood, manner, or vehicle for expressing an ethical imperative, to the detriment of the imperative itself. To avoid this it is necessary, in the formulation of each guideline, to be quite clear which ethical imperative is at stake. It is quite conceivable that one and the same guideline could be expressed in completely different words, concepts, and images for different communitieseven using the same language (such as English) which has developed as many varieties as there are identifiable groups using it.

At the level of application, the important thing again is to grasp the ethical imperative underlying the guideline, and cultural and circumstantial particulars will then do the work of "shaping" and "colouring" the guideline, without any need for further deliberate effort. If an ethical imperative cannot be identified in a guideline, then it may be a guideline but not an ethical guideline and complying with it can only be for non-ethical reasons, such as political or economic expediency or the necessity to obey a master.

In outline and very general terms this is, hopefully, the simple and clear conceptual model that I would recommend for the formulation and application of international ethical guidelines. Since the Nuremberg Code, and until the very recent revisions of the Helsinki and CIOMS guidelines, what had taken place in the formulation of "international" ethical guidelines were basically attempts to universalise and globalise a particularly powerful paradigm, a Western paradigm; even if it has much to recommend it and has also admittedly attempted to borrow and incorporate a few foreign ideas into its framework. The trend to involve other voices, perspectives, and cultures in the formulation of international ethical guidelines is one that needs encouraging and enhancing, in view of the fact that much of the so called developing world are also ex colonies of much of the developed world. Ex colonised peoples are liable to have suspicions and misgivings of projects, even apparently philanthropic ones, initiated and pushed by erstwhile colonising powers, because of post-colonial experiences tending to support the view that the process of decolonisation has not been completed beyond mere symbolism. Giving an increasing say to other communities, peoples, and cultures, and incorporating their views in international regulatory documents - as attempted, albeit in as yet a rather timid manner, in CIOMS 2002-is one way of helping to complete the process of decolonisation in a world increasingly becoming a global village.

\section{ACKNOWLEDGEMENTS}

This paper was written as the "practicum" part of the Johns HopkinsFogarty African Bioethics Fellowship Training Program. I am indebted to my fellowship advisors who read through each of the several drafts of this paper and provided me with provocative comments and suggestions, as well as important source materials. I am also thankful to the various conference audiences to whom I have been privileged to expose the ideas and arguments of this paper, for their stimulating responses. Lastly, I thank the Ministry of Higher Education and the University of Yaounde 1, Cameroon, for permitting me to benefit from the Hopkins-Fogarty Fellowship in 2002.

This work was supported in part by part of a grant (R25TW01604) from the Fogarty International Center, National Institutes of Health, DHHS, for Bioethics Training for Developing Country Professionals/Researchers

\section{REFERENCES}

1 Little MO. On Knowing the 'Why': Particularism and Moral Theory. Hastings Center Report 2001;31:32-40.

2 See Kant's rather terse reply to Benjamin Constant, "Concerning a Pretended Right to Lie from Motives of Humanity". Journal of Speculative Philosophy 1873;7: reprinted in Robert N. Beck, John B. Orr, Ethical Choice: A Case Study Approach New York: The Free Press, 1970:19-23.

3 Macklin RA. Defence of Fundamental Principles and Human Rights: A Reply to Robert Baker. Kennedy Institute of Ethics Journal 1999;8:403-22.

4 Toulmin S. The Tyranny of Principles. The Hastings Center Report (December), 1981:31-9.

5 Fidler DP. "Geographical Morality" Revisited: International Relations, International Law, and the Controversy over Placebo-Controlled HIV Clinical Trials in Developing Countries. Harvard International Law Journal 2001;42:300-54.

6 Wiredu K. Cultural Universals and Particulars: An African Perspective. Indiana University Press, 1996.

7 Macklin R. Bioethics, Vulnerability and Ethics. BIOETHICS. [in press].

8 Other issues. Developing World Bioethics 2002;1:92.

9 International Ethical Guidelines for Biomedical Research Involving Human Subjects. CIOMS, 1993:9

10 Human D. Why the World Medical Association Decided to Revise the Declaration of Helsinki. Unpublished conference address, 2001:3-4.

11 See reference 8: "Background Note".

12 IJsselmuiden C. Research Design as a Focal Point for Science and Ethics. Unpublished conference presentation, 2001.

13 See reference 11:8\&6.

14 Benatar SR, Singer PA. A new look at international research ethics. BMJ 2000;321:824-6. 